\title{
Facial pain from various sources-diagnoses and differential diagnoses
}

\section{Nelson Hendler*}

Former Assistant Professor of Neurosurgery, Johns Hopkins University School of Medicine; Former Associate Professor of Physiology, University of Maryland School of Dental Surgery; Past president-American Academy of Pain Management; Chief Operating Officer- Mensana Clinic Diagnostics, USA

The most common reason a patient seeks dental care is the sensation of pain in the teeth, jaw or face. While the assumption is that this pain is dental in origin, on occasion the pain may have a medical origin, either due to a medical disease, or a medical or neurological condition which manifest with pain in the teeth, jaw or face. Therefore, it is imperative that any dentist or oral surgeon be aware of these condition, to avoid misdiagnosis of the patient.

A group of from Johns Hopkins Hospital have published a series of reports, indicating that the misdiagnosis rate in chronic pain patients ranges from $40 \%$ to $80 \%$ [1-4]. For certain disorders, the misdiagnosis rate may be as high as $97 \%[5,6]$. The most common cause for misdiagnosis was failure to take a carful history, and ordering the wrong tests [7].

The other most common cause is a failure to consider a disorder. Essentially, this means if a physician does not consider a disease as a possibility, then he or she will not ask questions pertinent to that disease, and will fail to diagnosis it. Thus, it is imperative that a dentist or oral surgeon take a careful medical history.

One of the most important diagnostic consideration for any disease is the location of the pain. The Sensory Nerve Chart (Figure 1) shows nerves which cause pain to the face, teeth and jaw. Not only do many of the 12 cranial nerve (nerve which come directly off of the brain) have painful conditions, but the nerve roots (nerve which come off of the spinal cord) also can cause face pain.

Typically, cranial nerves branch into smaller and smaller nerves which provide sensory feedback from discrete anatomical location. However, in other situations, a most central pathology, like a tumor in the brain, can manifest as pain which seems to be of dental origin. The C2-3 root may refer pain to the angle of the jaw, or to an area behind the eye. The occipital nerve may give pain like a stripe up the back of the head. The facial and trigeminal nerve are the most common nerves involved in dental and facial pain.

The other factor to consider is the type of pain. Is the pain constant, or intermittent? If it is constant, this suggests a compressive, space occupying tumor. If it is intermittent, this suggests a position or metabolic process. Is the pain burning, sharp shooting pain, a throbbing pain, or a dull aching pain? Burning suggests damage to small, unmyelinated C fibers, which are susceptible to compression, infection, or chemical irritation. Sharp shooting pain suggests nerve compression. A throbbing pain suggests vascular compression. A dull aching pain is usually indicative of a slow and chronic process.

What was the onset of the pain? Sudden or slow and progressive?
A sudden onset of pain can relate to an accident, vascular event, or infarct. A slow, progress onset suggests that the origin of the pain is either due to infection, or a growth of a tumor.

A variety of medical problems present as dental problems, with pain either in the teeth and jaws. The most common of these is tempromandibular joint syndrome. This can manifest as damage to the ligaments and/or disc of the joint between the skull and the head of the jaw. The temporomandibular (TMJ) joint is a very unusual joint. It is

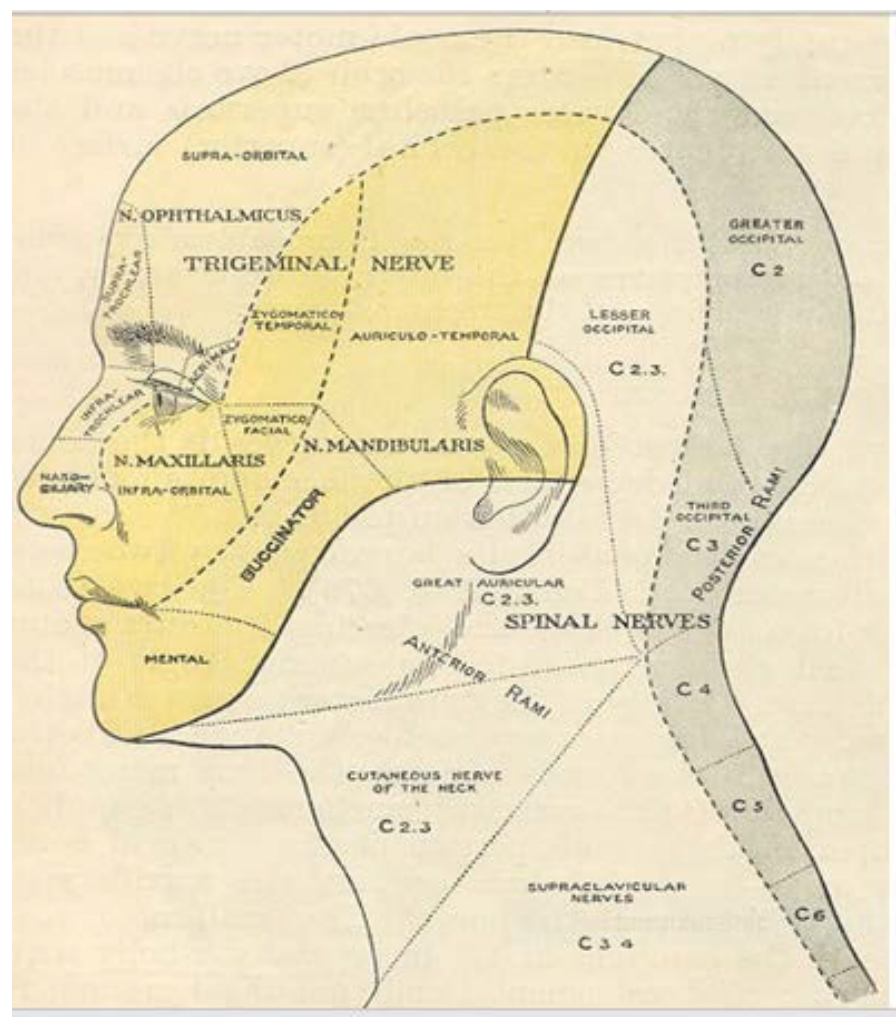

Figure 1. Note that the $\mathrm{C} 2-\mathrm{C} 3$ nerve root from the spinal cord has sensory projections on to the face, while cranial nerve (N 1-N12) sensory projections come from the brain directly.

Correspondence to: Nelson Hendler, MD, MS, Former Assistant Professor of Neurosurgery, Johns Hopkins University School of Medicine; Former Associate Professor of Physiology, University of Maryland School of Dental Surgery; Past president-American Academy of Pain Management; Chief Operating OfficerMensana Clinic Diagnostics, USA, E-mail: docnelse@aol.com

Received: May 22, 2017; Accepted: June 05, 2017; Published: June 08, 2017 
formed by the head of the condoyle of the jaw (mandible) inserting into a recess in the skull right beneath the temporal bone.

The jaw bone is held to the skull by ligaments which hold the jaw to the skull. These are the strong lateral temporomandibular ligaments and two weaker medial ligaments [8]. The nerves that supply the joint are the auriculotemporal and masse's tric branches of the third division of the trigeminal nerve [8] Irritation of these nerves produces pain in the jaw, cheek, and/or temple. There may be a central component. Typical symptoms are: facial pain, in the cheek, pain in the area of the joint, pain in the jaw line, ringing in the ear, dizziness, pain in the temple and clicks in the jaw on opening or closing the jaw [9] (Figure 2).

Recent evidence from Holland suggest that the origin of temporomandibular joint syndrome is not anxiety and stress as previously thought, but that TMJ may be a variant of an abnormal movement disorder, like dystonia, torticollis, Parkinson disease, or focal epilepsy. Dr. Peter Hamburger at Slotervaart Hospital in Amsterdam, The Netherlands, did studies on EEG abnormalities associated with EMG recording from the masseter muscles. He found antecedent EEG activity before the masseter muscle went into spasm. Preliminary studies with Topamax proved effective. (personal communication). There is even evidence that TMJ may have hereditary components [10].

In TMJ, either the disc or ligaments are torn, and this allow the condyle to move more than it should. Sometime the disc moves too, and gets trapped between the front of the fossa and the condyle, when the jaw is opened. This causes the click heard with TMJ, which is sort of like snapping your fingers.

Temporal arteritis (Giant cell arteritis) is one of the most serious facial pain problems, with potentials for stroke and/or blindness. The symptoms are frequent headaches, scalp tenderness, jaw pain and vision problems. Head pain and tenderness usually affects both temples. Associated with this is jaw pain when chewing or opening mouth wide, which usually can be confused with temporomandibular joint syndrome. However, when a patient has vision loss or double vision, particularly in people who also have jaw pain, you must think

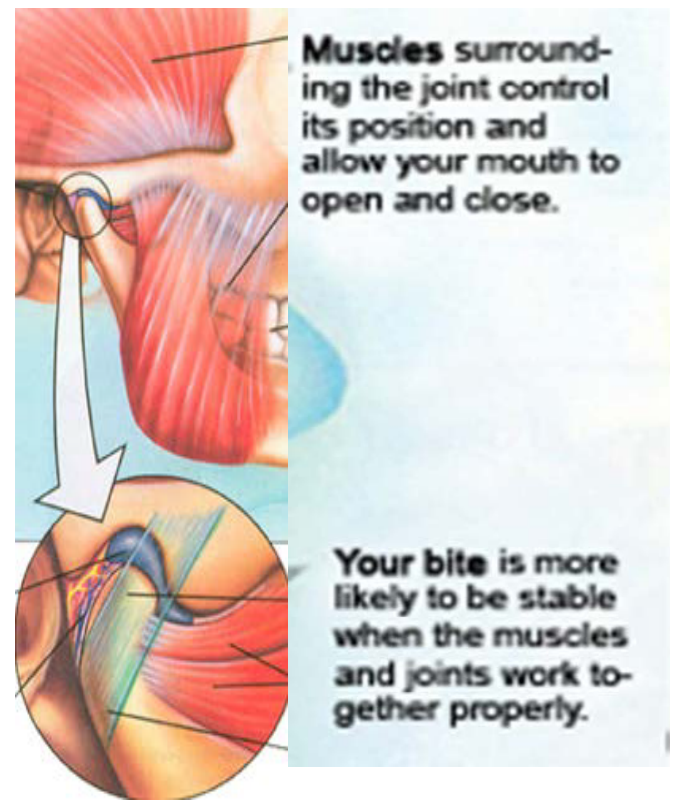

Figure 2. Ligaments are the tissue which hold bones together, while tendons attach muscle to the bone. the muscles move the bone. Muscles can get overstretched and spasm if the ligaments are damaged, and allow excessive movement of the bone. of temporal arteritis A sudden, loss of vision in one eye may become permanent, and may lead to stroke. About 50 percent of people with giant cell arteritis also have polymyalgia rheumatic (Figure 3).

The causes for temporal arteritis are well defined. The lining of arteries becomes inflamed. This causes them to swell. This swelling narrow the blood vessels, reducing the amount of blood, and therefore, oxygen and vital nutrients which reaches tissues in the body. Any large or medium-sized artery can be affected, but swelling most often occurs in the arteries located in the temples. The classic diagnostic feature of temporal arteritis is the observation that the temporal arteries are tender with a reduced pulse and a hard, cord-like feel and appearance. Blood test for sed rate and $\mathrm{C}$ reactive protein are almost always elevated. The sine qua non of diagnosis is biopsy of the temporal artery. The artery will often show inflammation that includes abnormally large cells, called giant cells. MRA, PET scan and Doppler or ultrasound also can help confirm the vascular nature of the disorder [11]. If the diagnosis of temporal arteritis is suspected, immediately start high dose prednisone to prevent vision loss, even before test results are available, and continue for one to two years. Also, it would be prudent to add methotrexate.

There are two main etiologies to the pain of trigeminal neuralgia. In both types, the pain can be constant, or intermittent, and follows the path of the sensory branches of the trigeminal nerve, into the forehead, along the cheek bone, or in the jaw line or any combination of the three [12].

One etiology is a congenital compression of the trigeminal nerve by the middle cerebral artery in the skull. This was well researched by Peter Janetta, MD and his team at Uni. of Pittsburgh [13]. Other researcher have found that compression of trigeminal nerve by loops of superior cerebellar artery or anterior inferior cerebellar artery $[14,15]$ (Figures 4 and 5).

When vascular compression causes trigeminal neuralgia, microvascular decompression of trigeminal nerve for facial pain is indicated. On the left is the external view of the posterior approach to the trigeminal nerve, and on the right is the what the surgeon actually sees. Note the relationship between the trigeminal nerve and the middle cerebral artery.

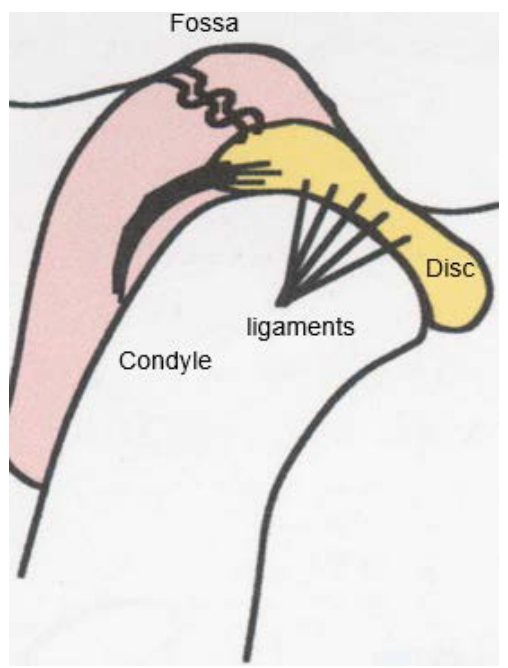

Figure 3. Below-in TMJ, either the disc or ligaments are torn, and this allows the condyle to move more than it should. Sometimes the disc moves too, and gets trapped between the front of the fossa and the condyle. When the jaw is opened. this causes the click heard with $\mathrm{TMJ}$, which is sort of like snapping your fingers. 

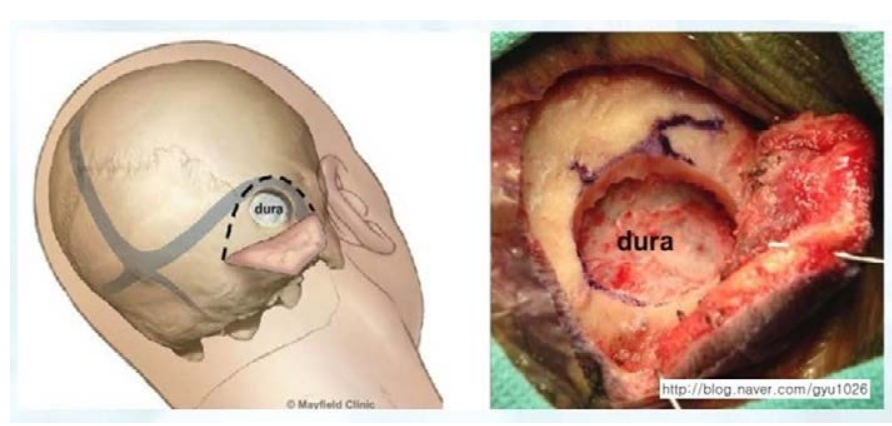

Figure 4. When vascular compression causes trigeminal neuralgia, microvascular decompression of trigeminal nerve for facial pain is indicated. On the left is the external view of the posterior approach to the trigeminal nerve, and on the right is the what the surgeon actually sees.

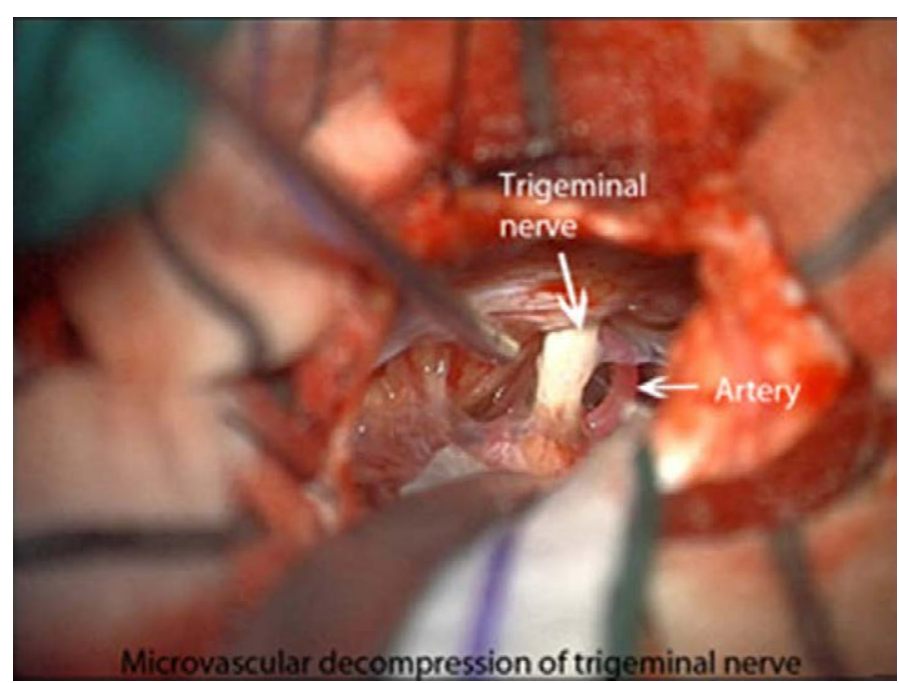

Figure 5. Note the relationship between the trigeminal nerve and the middle cerebral artery.

A second form of trigeminal neuralgia is a herpetic-like viral irritation of the trigeminal nerve [12]. Interestingly, the pain of trigeminal neuralgia from both sources is clinically the same. This is an example of "convergence," which is a single clinical manifestation due to several etiologies. The pain of both types of trigeminal neuralgia can be constant, or intermittent, and follows the path of the sensory branches of the trigeminal nerve, into the forehead, along the cheek bone, or in the jaw line or any combination of the three [12]. In both types of trigeminal neuralgia, the pain is sensitive to light touch, (hyperalgesia), and may occur in burst of pain, rather than being constant. The bursts of pain also occurs in both types of trigeminal neuralgia. In order to differentiate between the two forms of trigeminal neuralgia, an MRI of the brain, with vascular enhancement is useful. If there is no vessel compression, it is highly likely the cause of the pain is postherpetic. In addition to an MRI with vascular enhancement, an MRI of the brain, with thin-sections ( $2 \mathrm{~mm}$ slices), through the trigeminal nerve, and an MRA (Magnet resonance angiography) may be helpful in differentiating the post-viral etiology from the compression of the nerve by the blood vessel. Since there are two main etiologies to the pain of trigeminal neuralgia, there are two types of treatment. If the etiology is compression of the trigeminal nerve by the middle cerebral artery in the skull, then the treatment is intra-cranial decompression of the nerve, by inserting a sponge between the artery and the nerve [13]. If the etiology is due to a herpetic-like viral irritation of the trigeminal nerve, then the treatment is the use of medications [12]. Some people respond to low dose anti-depressants, but the most useful medication is an anti-convulsant, such as gaba-pentin (Neurontin), or Tegretol. Anti-viral drug, such as acyclovir will also help. If pharmacological intervention is not effective, surgical treatments including radiosurgery (mainly gamma knife therapy), radiofrequency (RF) of the Gasserian ganglion. RF is one of the most common procedures used to treat TN. Two RF approaches are commonly utilized: conventional radiofrequency (CRF) and pulsed radiofrequency (PRF) [16].

Very often, tooth pain is not from the teeth. Sinus infection irritating the medial branches of the facial nerve, mimic "toothache." Other 7th cranial nerve disorders involve diseases of the facial nerve or nuclei. The nerve may be involved intracranially, along its course through the petrous portion of the temporal bone, or along its extracranial course. Clinical manifestations include facial muscle weakness, loss of taste from the anterior tongue, or tooth pain. A sinus infection (sinusitis) or inflammation can cause a toothache - specifically in the upper rear teeth, which have roots close to the maxillary sinuses.

The diagnostic processes to clarify the diagnosis of 7 th nerve pain is as varied as the origins of the disorder. Indium 111 scan to look for infection in the sinus region, or roots of the teeth. This is more reliable than an anatomical test, such as a dental X-ray. An MRI of the brain to look for space occupying lesion in the brain, which could push on the $7^{\text {th }}$ nerve. Once the cause is thought to be infective, then obviously, antibiotics are used to combat the sinus infections or infected root. Anti-inflammatory and/or anticonvulsants drugs will not treat any disorder, but can be used to make a patient comfortable, until the source is identified and treated (Figure 6).

Eagles syndrome is characterized by a specific orofacial pain secondary to calcification of the stylohyoid ligament or elongated styloid process. In about $4 \%$ of general population an elongated styloid process occurs, but only about $4 \%$ of these patients are symptomatic, i.e. $4 \%$ of $4 \%$ or about one or two people per thousand. The stylo-hyoid bone is considered abnormal if it is greater than $25 \mathrm{~mm}$. in length [17]. The patient has a sensation of a foreign body localized at the tonsillar fossa, associated with a dull intermittent pain. Pain is usually worse with swallowing. A bony projection is usually palpable with bimanual trans-oral exploration. Basically, you put your finger in the mouth of the patient, down their throat, and push towards the outside, where the stylohyoid bone is. If this reproduces the pain, this confirms the diagnosis A lateral radiograph and a computed tomography scan of head and neck will show an elongated styloid process of at least $25 \mathrm{~mm}$ [17].

3D-CT of a very long stylo-hyoid bone....much longer than 25

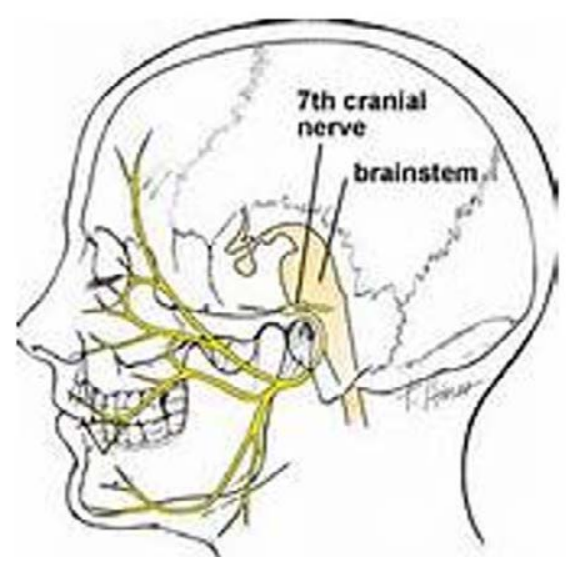

Figure 6. The various sensory projections of the 7th nerve. note sensory input to molars. 
mm. Follow the numbers 1,2,3 and 4 on both sides of the 3D-CT, and the is the stylo-hyoid bone, which is about $39 \mathrm{~mm}$ in this $3 \mathrm{D}-\mathrm{CT}$. The bone marked $\mathrm{H}$ is the hyoid bone. In this $3 \mathrm{D}-\mathrm{CT}$, the stylo-hyoid bone is so long that is spontaneously broke between 2 and 3 but it is still symptomatic, and causes pain with swallowing. Removal of the bone from the break to 4 on the 3D-CT will relieve symptoms (Figure 7).

Medical treatment represents the first choice. Use non-steroidal anti- inflammatory drugs. If this is not effective, then surgical resection of the styloid process may help (cutting off the tip). In the case of persistence of the complaint, surgery may be the only option. The symptoms may overlap with glossopharyngeal neuritis [17].

Sjogren syndrome is an autoimmune disorder in which the glands which produce tears and saliva are destroyed, causing dry mouth and dry eyes. Symptoms may include itching eyes, feeling that something is in the eye, difficulty swallowing, loss of sense of taste, thick saliva, mouth sores, many cavities in the teeth, and pain in the face, usually in the trigeminal or facial nerve [18]. Sjogren syndrome easily can be mistaken for trigeminal neuralgia. When there is unexplained pain in the face, the dentist should take a careful history. Ask about dry mouth, itchy eyes (SICCA complex, which is abnormal dryness of the mouth, eyes, or other mucous membranes), lots of cavities, or trouble swallowing [19]. Trigeminal sensory neuropathy can occur with Sjogren's and may be characterized by progressive sensory complaints on the face. They could start on one side and subsequently become bilateral (Steven Mandel, M.D., Clinical Professor of Neurology, Jefferson Medical College, personal communication). A variety laboratory studies, which include but are not limited to blood studies for SSA and SSB, ANA, sed rate, rheumatoid factor, (RF) and direct studies to the eye including the Schirmer test, corneal fluorescein staining, conjunctival lissamine green staining, and tear-film breakup time. Finally, lip biopsy is a useful method of diagnosing Sjogren's but it is not always reliable $[20,21]$. The treatment of Sjogren's syndrome involves conventional immunosuppressive therapies such as corticosteroids, which have been used in some patients with variable benefit. Current therapy for dryness is principally symptomatic. The greatest experience with treatment of Sjogren's syndrome is with rituximab, an anti-CD20 (expressed on a subset of B-cells) monoclonal antibody [22].

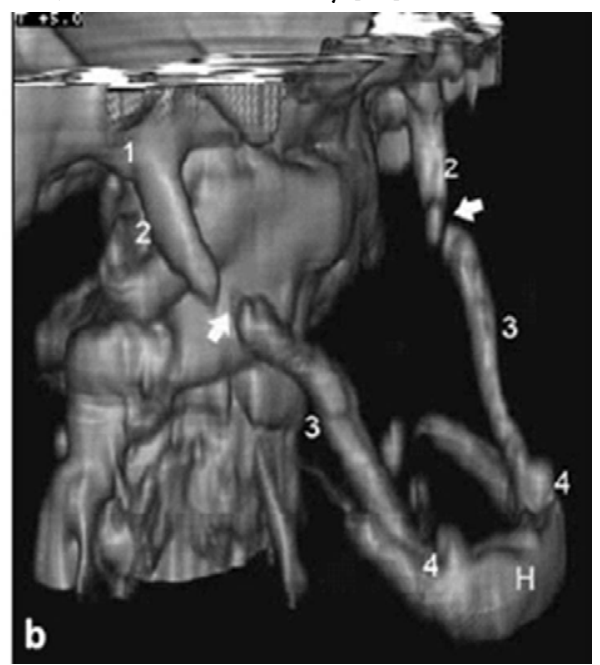

Figure 7. 3D-CT of a very long stylo-hyoid bone.... much longer than $25 \mathrm{MM}$. Follow the numbers 1,2,3 and 4 on both sides of the 3D-CT, and the is the stylo-hyoid bone, which is about $39 \mathrm{~mm}$ in this $3 \mathrm{D}-\mathrm{CT}$. The bone marked $\mathrm{h}$ is the hyoid bone. in this $3 \mathrm{D}-\mathrm{CT}$, the stylo-hyoid bone is so long that is spontaneously broke between 2 and 3 but it is still symptomatic, and causes pain with swallowing. Removal of the bone from the break to 4 on the $3 \mathrm{~d}-\mathrm{ct}$ will relieve symptoms.
Glossopharyngeal neuralgia is classically described as "stabbing," or "electrical shock-like" in nature. Episodes are typically brief, lasting a couple of seconds up to a few minutes and can be quite debilitating. They can occur in the tongue, throat, ear, and tonsils on one side of the head, but in rare cases may be bilateral. Painful attacks may occur spontaneously but are more often associated with a specific stimulus such as chewing, speaking, coughing, swallowing or laughing. The attacks can occur frequently throughout the day or once every couple of weeks and they can also disturb sleep [23]. As with other syndromes (glossopharyngeal neuralgia has many etiologies [23]. Glossopharyngeal neuralgia is typically caused by compression of the glossopharyngeal nerve. Compression of the nerve by an adjacent artery and/or vein can occur near the brainstem. Compression of the nerve more distally in the neck by an elongated styloid process (a bone in the neck) can cause this pain syndrome as well, and this is called Eagle syndrome. Glossopharyngeal neuralgia can be caused by a tumor or infection in the region of ninth cranial nerve or by multiple sclerosis [23].

The diagnosis of glossopharyngeal neuralgia is made on clinical grounds alone; no specific test can be done to prove the diagnosis in any given patient. Episodic ear and/or throat pain, often triggered by touching the palate or tonsil is strongly suggestive of the disorder. High resolution MRI or CT imaging of the brainstem may reveal vascular compression, tumors, or demyelinating lesions involving the ninth cranial nerve. High resolution CT scanning of the neck can reveal an elongated styloid process, suggestive of Eagle syndrome. Pain that distinctly involves the ear, the rear $1 / 3$ of the tongue and/or throat is the classic pain distribution seen in glossopharyngeal neuralgia (ninth cranial nerve or IX N [23]

Most treatment for Glossopharyngeal Neuralgia is pharmacological. Anticonvulsant medications, including oxcarbazepine, carbamazepine, gabapentin, and pregabalin Some antidepressants can also be helpful either alone or in combination with the seizure medications. If medications fail, surgical intervention is indicated. The first-choice for treatment is microvascular decompression (MVD), as it has the highest initial and long-term success rates. Patients unable to tolerate such an operation may consider one of the many less invasive options including Gamma Knife radiosurgery (Table 1). Eagles syndrome, TMJ and Trigeminal neuralgia can co-exist with glossopharyngeal neuritis, and mask the diagnosis, or be mistaken for it $[16,17,19,22,23]$.

While the disorders mentioned in this article are no means a comprehensive, this paper should provide a primer for any dentist wishing to consider the differential diagnoses of medical problems which may present as dental problems. The cornerstone of any dental diagnosis is obtaining a careful history. However, this often is a timeconsuming process. Therefore, a number of clinicians have resorted to self-administered questionnaires. Unfortunately, the questionnaires completed by patient are very rarely reviewed by the dentist or physician. In order to, combat the time constraints in obtaining a careful history, and an accurate interpretation of the answers, a team of doctors from Johns Hopkins Hospital have developed an Internetbased expert diagnostic system for chronic pain, available in either

Table 1. Overlap of clinical symptoms

\begin{tabular}{|l|l|}
\hline Jaw pain & TMJ, temporal arteritis, trigeminal neuralagia \\
\hline Temple pain & TMJ, temporal arteritis, trigeminal neuralagia, facial nerve neuralgia \\
\hline Tooth pain & Sinusitis, trigeminal neuralagia, facial nerve neuralgia \\
\hline Swallowing pain & Eagles syndrome, Glossopharyngeal neuritis \\
\hline Cheek pain & Sjogren's syndrome, trigeminal neuralagia \\
\hline
\end{tabular}


English or Spanish. When a patient completes this questionnaire, a proprietary computer program analyzes the answers, using Bayesian analysis. Within five minutes after a patient completes the questionnaire, the clinician receives a list of diagnoses, and differential diagnoses, which have a $96 \%$ correlation with diagnoses of Johns Hopkins hospital doctors. This questionnaire is available at www. MarylandClinicalDiagnostics.com [24].

\section{References}

1. Long D, Davis R, Speed W, Hendler N (2006) Fusion for occult post-traumatic cervical facet injury. Neurosurgery quarterly 16: 129-134.

2. Hendler N (2002) Differential diagnosis of complex regional pain syndrome. Pan Arab Journal of Neurosurgery 1-9.

3. Hendler N, Bergson C, Morrison C (1996) Overlooked physical diagnoses in chronic pain patients involved in litigation, Part 2. The addition of MRI, nerve blocks, 3-D CT, and qualitative flow meter. Psychosomatics 37: 509-517. [Crossref]

4. Dellon AL, Andonian E, Rosson GD (2009) CRPS of the upper or lower extremity: surgical treatment outcomes. J Brachial Plex Peripher Nerve Inj 4: 1. [Crossref]

5. Hendler N (2005) Overlooked diagnosis in electric shock and lightning strike survivors. J Occup Environ Med 47: 796-805. [Crossref]

6. Nelson Hendler, Thomas Romano (2016) Fibromyalgia over-diagnosed 97\% of the time: chronic pain due to thoracic outlet syndrome, acromo-clavicular joint syndrome, disrupted disc, nerve entrapments, facet syndrome and other disorders mistakenly called fibromyalgia. J Anesth Pain Med 1: 1-7.

7. Laura Landro (2013) The biggest mistake doctors make. The Wall Street Journal.

8. Guralnick W, Kaban LB, Merrill RG (1978) Temporomandibular-joint afflictions. $N$ Engl J Med 299: 123-129. [Crossref]

9. Gelb H (1978) TMJ Syndrome: The tell-tale click, Behavioral Medicine.

10. Visscher CM, Lobbezoo F (2015) TMD pain is partly heritable. A systematic review of family studies and genetic association studies. J Oral Rehabil 42: 386-399. [Crossref]

11. Czihal M, Lottspeich C, Hoffmann U (2017) Ultrasound imaging in the diagnosis of large vessel vasculitis. Vasa 23: 1-13. [Crossref]

12. Empting, L, Hendler, N, Kolodny, L, Kraus, H (1990) Tips on hard to manage pain syndromes. Patient Care 26-46.

13. Sekula RF, Marchan EM, Fletcher LH, Casey KF, Jannetta PJ (2008) Microvascular decompression for trigeminal neuralgia in elderly patients. J Neurosurg 108: 689-691. [Crossref]

14. Jha P (2017) Trigeminal neuralgia: vascular compression, Radiopedia, https:// radiopaedia.org/articles/trigeminal-neuralgia.

15. Di Muzio B (2017). Trigeminal neuralgia, Radiopedia, https://radiopaedia.org/articles/ trigeminal-neuralgia.

16. Guo J, Dong X, Zhao X (2016) Treatment of trigeminal neuralgia by radiofrequency of the Gasserian ganglion. Rev Neurosci 27: 739-743.

17. Casale M, Rinaldi V, Quattrocchi C, Bressi F, Vincenzi B, et al. (2008) Atypical chronic head and neck pain: don't forget Eagle's syndrome. Eur Rev Med Pharmacol Sci 12: 131-133. [Crossref]

18. Kruszka P, O’Brian RJ (2009) Diagnosis and management of Sjögren syndrome. $A m$ Fam Physician 79: 465-470. [Crossref]

19. Wu AJ (2008) Optimizing dry mouth treatment for individuals with Sjogren's syndrome. Rheum Dis Clin North Am 34: 1001-1010. [Crossref]

20. Yang Z, Liang Y, Zhong R (2012) Is identification of anti-SSA and/or -SSB antibodies necessary in serum samples referred for antinuclear antibodies testing? J Clin Lab Anal 26: 447-451. [Crossref]

21. Liew MS, Zhang M, Kim E, Akpek EK (2012) Prevalence and predictors of Sjogren's syndrome in a prospective cohort of patients with aqueous-deficient dry eye. $\mathrm{Br} J$ Ophthalmol 96: 1498-1503. [Crossref]

22. Bowman S, Barone F (2012) Biologic treatments in Sjogren's syndrome. Presse Med 41: e495-509. [Crossref]

23. http://www.columbianeurosurgery.org/conditions/glossopharyngeal-neuralgia/.

24. Hendler N, Berzoksky C, Davis RJ (2007) Comparison of clinical diagnoses versus computerized test diagnoses using the mensana clinic diagnostic paradigm (expert system) for diagnosing chronic pain in the neck, back and limbs. Pan Arab Journal of Neurosurgery 8-17.

Copyright: (C2017 Hendler N. This is an open-access article distributed under the terms of the Creative Commons Attribution License, which permits unrestricted use, distribution, and reproduction in any medium, provided the original author and source are credited. 